\title{
Enstrophy dynamics of stochastically forced large-scale geophysical flows
}

Cite as: Journal of Mathematical Physics 43, 2616 (2002); https://doi.org/10.1063/1.1459755

Submitted: 29 June 2001 . Accepted: 24 January 2002 . Published Online: 18 April 2002

Dirk Blömker, Jinqiao Duan, and Thomas Wanner

\section{Don't let your writing} keep you from getting published!

AIDAthor Services 


\title{
Enstrophy dynamics of stochastically forced large-scale geophysical flows
}

\author{
Dirk Blömker ${ }^{\mathrm{a})}$ \\ Institut für Mathematik, Universität Augsburg 86135 Augsburg, Germany \\ Jinqiao Duan \\ Department of Applied Mathematics, Illinois Institute of Technology, \\ Chicago, Illinois 60616 \\ Thomas Wanner \\ Department of Mathematics and Statistics, University of Maryland, \\ Baltimore County, Baltimore, Maryland 21250
}

(Received 29 June 2001; accepted for publication 24 January 2002)

Enstrophy is an averaged measure of fluid vorticity. This quantity is particularly important in rotating geophysical flows. We investigate the dynamical evolution of enstrophy for large-scale quasi-geostrophic flows under random wind forcing. We obtain upper bounds on the enstrophy, as well as results establishing its Hölder continuity and describing the small-time asymptotics. (C) 2002 American Institute of Physics. [DOI: 10.1063/1.1459755]

\section{INTRODUCTION}

Randomness is ubiquitous in fluid systems. Macroscopic partial differential equation models for fluid flows contain such randomness as stochastic forcing, uncertain parameters, random sources, and random boundary conditions.

There has been active recent research on stochastic approaches to geophysical flows ${ }^{1-5}$ and numerical simulations of stochastically forced geophysical flows. ${ }^{6-10}$ It is generally understood that random fluctuations can have delicate impact on geophysical fluid dynamics. ${ }^{1,3,6,7,11}$

A class of large-scale geophysical flows under random forcing are modeled by the quasigeostrophic equation: ${ }^{1}$

$$
\Delta \psi_{t}+J(\psi, \Delta \psi)+\beta \psi_{x}=\nu \Delta^{2} \psi-r \Delta \psi+\dot{W},
$$

where $\psi(x, y, t)$ is the stream function $\left(\psi_{x}:=\partial_{x} \psi\right), \beta \geqslant 0$ is the meridional gradient of the Coriolis parameter, $\nu>0$ is the viscous dissipation constant, $r>0$ is the Ekman dissipation constant, and $W(x, y, t)$ is a space-time Wiener process to be defined below on a probability space $(\Omega, \mathcal{A}, \mathbb{P})$. Moreover, $J(f, g)=f_{x} g_{y}-f_{y} g_{x}$ denotes the Jacobian operator. The generalized time derivative $\dot{W}$ models the noisy wind forcing.

Introducing $\omega(x, y, t)=\Delta \psi(x, y, t)$, Eq. (1) can be rewritten in the form

$$
\omega_{t}+J(\psi, \omega)+\beta \psi_{x}=\nu \Delta \omega-r \omega+\dot{W},
$$

where $(x, y) \in D$ and $D \subset \mathrm{R}^{2}$ denotes a bounded domain with sufficiently smooth boundary. The boundary conditions are no normal flow $(\psi=0$ on $\partial D)$ and free-slip $(\omega=0$ on $\partial D)$ as in Pedlosky (Ref. 12, p. 34) or in Dymnikov and Kazantsev: ${ }^{13}$

$$
\psi=\omega=0 \text { on } \partial D .
$$

An appropriate initial condition $\omega(0)$ is also imposed.

The mean enstrophy for a fluid flow is half the squared mean-square norm of the vorticity, ${ }^{14,15}$ i.e., we have

${ }^{a)}$ Present address: Institut für Mathematik, RWTH Aachen, D-52056 Aachen, Germany. 


$$
\operatorname{Ens}(t)=\frac{1}{2} \cdot \mathbb{E} \int_{D}|\omega(x, y, t)|^{2} d(x, y) .
$$

The enstrophy $\operatorname{Ens}(t)$ is an averaged measure of fluid vorticity $\omega(t)$. In this article, we discuss the time evolution of the enstrophy. We present results which establish upper bounds on the enstrophy, as well as results on Hölder continuity and small-time asymptotics for Ens $(t)$. These results are contained in Secs. III, IV, and V, respectively. The mathematical framework for our discussion is described in Sec. II.

\section{MATHEMATICAL FRAMEWORK}

As it stands, the stochastic quasi-geostrophic equation (2) still has to be given a mathematically precise meaning. This can be accomplished using the framework of stochastic partial differential equations, as described, for example, in Ref. 16. In our situation, we formally rewrite (2) in the Ito formulation

$$
d \omega=\left(\nu \Delta \omega-r \omega-\beta \psi_{x}-J(\psi, \omega)\right) d t+d W .
$$

In the following we use the abbreviations $L^{2}=L^{2}(D), L^{\infty}=L^{\infty}(D), H_{0}^{k}=H_{0}^{k}(D), H^{k}=H^{k}(D)$, $0<k<\infty$, for the standard Sobolev spaces. Let $\langle\cdot, \cdot\rangle$ and $\|\cdot\|$ denote the standard scalar product and norm in $L^{2}$, respectively. Moreover, the norms for $H_{0}^{k}$ and $L^{\infty}$ are denoted by $\|\cdot\|_{H^{k}}$ and $\|\cdot\|_{\infty}$, respectively. Due to the Poincaré inequality (Ref. 17 p. 164), the expression $\|\Delta \cdot\|$ is an equivalent norm for $H_{0}^{2}$. It is well-known that the operator $A=\nu \Delta: L^{2} \rightarrow L^{2}$ with domain $D(A)$ $=H^{2} \cap H_{0}^{1}$ is self-adjoint. Note that $A$ generates an analytic semigroup $S(t)$ on $L^{2} .{ }^{18}$ The spectrum of $A$ consists of eigenvalues $0>\lambda_{1}>\lambda_{2} \geqslant \lambda_{3} \geqslant \cdots$ with corresponding normalized eigenfunctions $\varphi_{1}, \varphi_{2}, \ldots$. The set of these eigenfunctions is complete in $L^{2}$. For example, for the square domain $D=(0,1) \times(0,1)$ the eigenvalues are given by $-\nu\left(m^{2}+n^{2}\right) \pi^{2}$ for $m, n \in \mathbb{N}$, and the associated eigenfunctions are suitable multiples of $\sin (m \pi x) \sin (n \pi y)$.

Now we can define an appropriate class of Wiener processes $W$. Let $\beta_{k}, k \in \mathbb{N}$, denote a family of independent real-valued standard Brownian motions. Furthermore, choose positive constants $\mu_{k}, k \in \mathbb{N}$, such that

$$
\sum_{k=1}^{\infty} \frac{\mu_{k}^{2}}{\left|\lambda_{k}\right|^{1-\theta}}<\infty
$$

for some $0<\theta<1$. Then we consider the Wiener process $W$ defined by

$$
W(t):=\sum_{k=1}^{\infty} \mu_{k} \cdot \beta_{k}(t) \cdot \varphi_{k}, \quad t \geqslant 0
$$

Note that we explicitly allow Wiener processes $W$ whose covariance operator is not of trace class, i.e., for which $\sum_{k=1}^{\infty} \mu_{k}^{2}=\infty$.

For the domain $D$ we basically assume that the eigenfunctions $\varphi_{k}$ of $A$ satisfy

$$
\begin{gathered}
\varphi_{k} \in C_{0}(\bar{D}), \quad\left|\varphi_{k}(x, y)\right| \leqslant C, \\
\left|\nabla \varphi_{k}(x, y)\right| \leqslant C \sqrt{\left|\lambda_{k}\right|},
\end{gathered}
$$

for all $(x, y) \in D$ and $k \in \mathbb{N}$, where $C>0$ denotes a constant which depends only on $D$. Domains $D$ which satisfy these conditions include rectangular domains, as well as equilateral triangles. Unfortunately, there are many domains for which they are violated. See, for example, Ref. 19. However, in this paper it is conjectured that in (7) one generally should expect an upper bound which is logarithmic in $\left|\lambda_{k}\right|$. Even though our results remain valid in this situation, we will assume the above stronger condition. 
Under the above assumptions, Theorem 5.2.9 in Ref. 20 guarantees that the stochastic convolution

$$
W_{A}(t)=\int_{0}^{t} S(t-s) d W(s), \quad t>0,
$$

has a continuous version with values in $C_{0}(D)$, the Banach space of continuous functions satisfying zero Dirichlet boundary conditions on $D$. To be more precise, $W_{A}$ has a version which is even Hölder continuous with some small exponent, which depends on the asymptotic behavior of the coefficients $\mu_{k}$.

If we define the nonlinear operator $F$ by $F(\omega)=-r \omega-\beta \psi_{x}-J(\psi, \omega)$, then (4) can be rewritten as the abstract evolution equation together with initial condition

$$
\begin{gathered}
d \omega=(A \omega+F(\omega)) d t+d W, \\
\omega(0)=\omega_{0} .
\end{gathered}
$$

For technical reasons we translate the operator $A$ by a suitable multiple of the identity. Consider a constant $\alpha \geqslant 0$ which will be chosen later on, usually sufficiently large. Defining $A_{\alpha}:=A-\alpha I$, we get the initial value problem

$$
\begin{gathered}
d \omega=\left(A_{\alpha} \omega+F(\omega)+\alpha \omega\right) d t+d W, \\
\omega(0)=\omega_{0}
\end{gathered}
$$

or in mild (integral) form

$$
\omega(t)=S_{\alpha}(t) \omega_{0}+\int_{0}^{t} S_{\alpha}(t-s)(F(\omega(s))+\alpha \omega(s)) d s+W_{A_{\alpha}}(t),
$$

where the analytic semigroup $S_{\alpha}$ is given by $S_{\alpha}(t)=e^{-t \alpha} \cdot S(t)$ for $t>0$ and the stochastic convolution $W_{A_{\alpha}}(t)$ is defined as in (8) with the semigroup $S$ replaced by $S_{\alpha}$. Finally, let $U:=\omega$ $-W_{A_{\alpha}}$. Then $U$ is the weak solution of

$$
\begin{gathered}
\partial_{t} U=A U+F(U+V)+\alpha V, \\
U(0)=\omega_{0},
\end{gathered}
$$

where we use the abbreviation $V:=W_{A_{\alpha}}$. Notice that both $U$ and $V$ depend on $\alpha$.

\section{ENSTROPHY ESTIMATE: UPPER BOUNDS}

We begin by establishing upper bounds on the time evolution of the enstrophy Ens $(t)$ $=\mathbb{E}\|\omega(t)\|^{2} / 2$. Improving the a priori estimate of Ref. 5 Sec. 3, we obtain

$$
\begin{aligned}
\frac{1}{2} \cdot \frac{d}{d t}\|U(t)\|^{2} \leqslant & \|\nabla U(t)\|^{2} \cdot(\varepsilon-\nu) \\
& +\|U(t)\|^{2} \cdot\left(\left(\varepsilon-r+c_{1} \beta\right)+C \cdot\|V\|_{\infty} \cdot\left(1+C_{\varepsilon} \cdot\|V\|_{\infty}\right)\right) \\
& +C_{\varepsilon} \cdot\left(r+\beta+\alpha^{2}\right) \cdot\|V\|_{\infty}^{2}+C \cdot\|V\|_{\infty}^{3}+C_{\varepsilon} \cdot\|V\|_{\infty}^{4},
\end{aligned}
$$

where $C$ denotes a generic constant which depends only on $D$, and whose specific value may change from line to line. Similarly, $C_{\varepsilon}$ denotes a generic constant which depends only on $D$ and 
$\varepsilon$, where $\varepsilon>0$ is some arbitrarily small number. The constant $c_{1}$ denotes the optimal constant in the Poincare inequality $\|U\| \leqslant c_{1}\|\nabla U\|$ for mean zero functions $U$. For $\varepsilon<\nu$ the improved a priori estimate immediately yields the following lemma.

Lemma 1: For any $\gamma>-\nu \cdot c_{1}^{-2}-r+c_{1} \cdot \beta$ there exist constants depending only on $\gamma, D, \beta$, and $r$ which are all denoted by $C$ such that

$$
\frac{d}{d t}\|U(t)\|^{2} \leqslant A(t)\|U(t)\|^{2}+B(t), \quad t \geqslant 0,
$$

with

$$
\begin{gathered}
A(t)=2 \gamma+C \cdot\left(\|V\|_{\infty}+\|V\|_{\infty}^{2}\right), \\
B(t)=C \cdot\left(\left(1+\alpha^{2}\right) \cdot\|V\|_{\infty}^{2}+\|V\|_{\infty}^{4}\right)>0 .
\end{gathered}
$$

Together with Theorem I.6.1 in Ref. 21 this yields

$$
\|U(t)\|^{2} \leqslant\left\|\omega_{0}\right\|^{2} \cdot e^{\int_{0}^{t} A(s) d s}+\int_{0}^{t} B(s) e^{\int_{s}^{t} A(\tau) d \tau} d s, \quad t \geqslant 0 .
$$

Suppose for simplicity that $\omega_{0}$ and $W$ are stochastically independent. It is possible to drop this assumption in this section, but in this case we additionally need $\mathbb{E}\left\|\omega_{0}\right\|^{2+\delta}<\infty$ for some small $\delta>0$.

The critical term for taking the expectation in (14) is the squared $L^{\infty}$-norm of $V=W_{A_{\alpha}}$ in the exponent, which is in general not finite. To complicate matters further, the $L^{\infty}$-norm in the exponent cannot easily be dealt with, since we do not have a Hilbert space structure.

For our situation we will improve on some ideas of Ref. 22. Using Fernique's Theorem (Ref. 16, Theorem 2.6) we get that $\mathbb{P}\left(t\|V(\tau)\|_{\infty}^{2}>r^{2}\right) \leqslant 1 /\left(1+e^{1+32 \lambda r^{2}}\right)$ implies $\mathbb{E}\left(e^{\lambda t\|V(\tau)\|_{\infty}^{2}}\right) \leqslant e^{16 \lambda r^{2}}$ $+e^{2} /\left(e^{2}-1\right)$ for any $t, \tau, r, \lambda>0$. Hence, by Jensen's inequality

$$
\mathbb{E}\left(e^{\lambda \int_{0}^{t}\|V(\tau)\|_{\infty}^{2} d \tau}\right) \leqslant \frac{1}{t} \cdot \int_{0}^{t} \mathbb{E}\left(e^{\lambda t\|V(\tau)\|_{\infty}^{2}}\right) d \tau \leqslant C_{\lambda},
$$

provided $\mathrm{P}\left(t\|V(\tau)\|_{\infty}^{2}>1\right) \leqslant 1 /\left(1+e^{1+32 \lambda}\right)$ for any $\tau \leqslant t$. The latter inequality follows immediately from Chebychev's inequality, provided we have

$$
t \cdot \mathbb{E}\|V(\tau)\|_{\infty}^{2} \leqslant \frac{1}{1+e^{1+32 \lambda}}
$$

for any $\tau \leqslant t$. The following lemma is proven similarly as Ref. 16, Theorem 5.20.

Lemma 2: For any $p \geqslant 1$ and any sufficiently small $\theta>0$ there exists a constant $C$ which depends only on $p, \theta$, and $D$ such that for any $\tau \geqslant 0$

$$
\mathbb{E}\|V(\tau)\|_{\infty}^{2 p} \leqslant C \cdot \underbrace{\left(\sum_{k=1}^{\infty} \frac{\mu_{k}^{2}}{\alpha-\lambda_{k}} \cdot\left|\lambda_{k}\right|^{\theta}\right)^{p}}_{=: \varphi(\alpha)^{p}} .
$$

We remark that the assumption (7) on the eigenfunctions is essential for the proof of this lemma. Notice also that the series in (16) is finite according to (5). Lemma 2 implies that (15) is satisfied for any $t \leqslant C / \varphi(\alpha)$. It is now straightforward to verify that

$$
\mathbb{E} e^{m \int_{s}^{t} A(\tau) d \tau} \leqslant C \cdot e^{m(t-s) 2 \gamma}
$$


for any $s \leqslant t \leqslant C / \varphi(\alpha)$ and $m=1,2$, where $\gamma$ and $A(t)$ were defined in Lemma 1. Moreover, Lemma 2 implies for $B(t)$ in (13)

$$
\left(\mathbb{E} B(t)^{2}\right)^{1 / 2} \leqslant C \cdot\left(\left(1+\alpha^{2}\right) \cdot \varphi(\alpha)+\varphi(\alpha)^{2}\right)
$$

for any $t>0$. We finally obtain from (14) that

$$
\begin{aligned}
\mathbb{E}\|U(t)\|^{2} & \leqslant \mathbb{E}\left(\left\|\omega_{0}\right\|^{2} \cdot e^{\int_{0}^{t} A(s) d s}+\int_{0}^{t} B(s) e^{\int_{s}^{t} A(\tau) d \tau} d s\right) \\
& \leqslant \mathbb{E}\left\|\omega_{0}\right\|^{2} \cdot \mathbb{E} e^{\int_{0}^{t} A(s) d s}+\int_{0}^{t}\left(\mathbb{E} B(s)^{2}\right)^{1 / 2}\left(\mathbb{E} e^{2 \int_{s}^{t} A(\tau) d \tau}\right)^{1 / 2} d s \\
& \leqslant C \cdot \mathbb{E}\left\|\omega_{0}\right\|^{2} \cdot e^{2 \gamma t}+C \cdot\left(\left(1+\alpha^{2}\right) \cdot \varphi(\alpha)+\varphi(\alpha)^{2}\right) \cdot \int_{0}^{t} e^{2 \gamma \tau} d \tau
\end{aligned}
$$

for any $t \leqslant C / \varphi(\alpha)$. Using $\mathbb{E}\|\omega\|^{2} \leqslant 2 \mathbb{E}\|U\|^{2}+2 \mathbb{E}\|V\|^{2}$, this immediately implies the following theorem on upper bounds for the enstrophy.

Theorem 1 (upper bound): Suppose that $\omega_{0}$ and $W$ are stochastically independent and that $\mathbb{E}\left\|\omega_{0}\right\|^{2}<\infty$. Moreover, let $\gamma>-\nu \cdot c_{1}^{-2}-r+c_{1} \cdot \beta$. Then $\mathrm{Ens} \in L^{\infty}([0, T])$ for any $T>0$. More precisely,

$$
\begin{aligned}
\operatorname{Ens}(t) \leqslant & C \cdot \mathbb{E}\left\|\omega_{0}\right\|^{2} \cdot e^{2 \gamma t}+C \cdot \varphi(\alpha) \\
& +C \cdot\left(\left(1+\alpha^{2}\right) \cdot \varphi(\alpha)+\varphi(\alpha)^{2}\right) \cdot \int_{0}^{t} e^{2 \gamma \tau} d \tau
\end{aligned}
$$

for any $t \leqslant C / \varphi(\alpha)$, with constants $C$ independent of $t, \alpha$, and $\omega_{0}$.

Remark 1: It can be shown that for any choice of $p \geqslant 1$ similar bounds hold for $\operatorname{Esup}_{\tau \in[0, t]}\|U(\tau)\|^{2 p}$, provided both $\mathbb{E}\left\|\omega_{0}\right\|^{2 p}<\infty$ and $t \leqslant C / \varphi(\alpha)$.

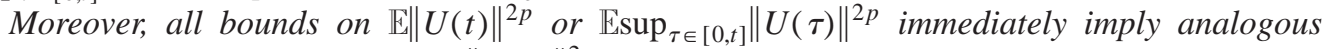
bounds on $\operatorname{Ens}(t)$ or $\mathbb{E s u p}_{\tau \in[0, t]}\|\omega(\tau)\|^{2 p}$. For this one has to employ estimates for $\operatorname{Esup}_{\tau \in[0, t]}\left\|W_{A_{\alpha}}(\tau)\right\|^{2 p}$ which can be obtained for example as in Ref. 23, Corollary 2.3.

In order to obtain a bound for $t \rightarrow \infty$, we note that for $\alpha \rightarrow \infty$ one obviously has $\varphi(\alpha) \rightarrow 0$. However, the rate of convergence is essential. To this end, we distinguish two cases. If we suppose that $\sum_{k=1}^{\infty} \mu_{k}^{2} \cdot\left|\lambda_{k}\right|^{\theta}<\infty$ for some $\theta>0$, then the estimate $\varphi(\alpha) \leqslant \sum_{k=1}^{\infty} \mu_{k}^{2} \cdot\left|\lambda_{k}\right|^{\theta} / \alpha$ is immediateand choosing $\alpha$ proportional to $t$ in Theorem 1 furnishes

$$
\text { Ens }(t) \leqslant C \cdot\left(\mathbb{E}\left\|\omega_{0}\right\|^{2} \cdot e^{2 \gamma t}+t \cdot \int_{0}^{t} e^{2 \gamma \tau} d \tau+1\right) \quad \text { for all } t \geqslant 0 .
$$

If, on the other hand, $\Sigma_{k=1}^{\infty} \mu_{k}^{2} \cdot\left|\lambda_{k}\right|^{\theta}=\infty$, we additionally assume $\mu_{k}^{2} \leqslant C k^{-\mu}$ for some $\mu \in(\theta, 1]$, with arbitrarily small $\theta$ defined in Lemma 2. (For $\mu>1$ we can always find some small $\theta$ such that the first case applies.) Using the fact that $\left|\lambda_{k}\right| \sim C k$ for $k \rightarrow \infty$ (cf. Ref. 24) we obtain

$$
\begin{aligned}
\varphi(\alpha) \leqslant C \cdot \sum_{k=1}^{\infty} \frac{k^{-\mu}}{c k+\alpha} \cdot k^{\theta} & \leqslant C \cdot \int_{0}^{\infty} \frac{k^{\theta-\mu}}{c k+\alpha} d k \\
& =C \cdot \alpha^{\theta-\mu} \cdot \int_{0}^{\infty} \frac{\tau^{\theta-\mu}}{c \tau+1} d \tau .
\end{aligned}
$$

Choosing $\alpha^{\mu-\theta}$ proportional to $t$ in Theorem 1, we derive

$$
\operatorname{Ens}(t) \leqslant C \cdot\left(\mathbb{E}\left\|\omega_{0}\right\|^{2} \cdot e^{2 \gamma t}+t^{2 /(\mu-\theta)-1} \cdot \int_{0}^{t} e^{2 \gamma \tau} d \tau+1\right) \quad \text { for all } t \geqslant 0
$$


Notice that $2 /(\mu-\theta)-1>1$. We have proved the following result.

Theorem 2 (global upper bound): Assume again that $\omega_{0}$ and $W$ are stochastically independent, that $\mathbb{E}\left\|\omega_{0}\right\|^{2}<\infty$, and let $\gamma>-\nu \cdot c_{1}^{-2}-r+c_{1} \cdot \beta$. Then the following holds.

(a) If $\mu_{k}^{2} \leqslant C k^{-\mu}$ for some $\mu \in(0,1]$, we can find a $\tilde{\mu} \in(0, \mu)$ such that

$$
\operatorname{Ens}(t) \leqslant C \cdot\left(\mathbb{E}\left\|\omega_{0}\right\|^{2} \cdot e^{2 \gamma t}+t^{(2-\tilde{\mu}) / \tilde{\mu}} \cdot \int_{0}^{t} e^{2 \gamma \tau} d \tau+1\right) \quad \text { for all } t \geqslant 0
$$

(b) If $\sum_{k=1}^{\infty} \mu_{k}^{2} \cdot\left|\lambda_{k}\right|^{\theta}<\infty$ for some $\theta>0$, then

$$
\operatorname{Ens}(t) \leqslant C \cdot\left(\mathbb{E}\left\|\omega_{0}\right\|^{2} \cdot e^{2 \gamma t}+t \cdot \int_{0}^{t} e^{2 \gamma \tau} d \tau+1\right) \quad \text { for all } t \geqslant 0 .
$$

The constants $C$ are independent of $t$ and $\omega_{0}$, but they can depend on $\theta, \gamma, \mu, \tilde{\mu}$, the domain $D$, or the coefficients in (1).

Remark 2: If $-\nu \cdot c_{1}^{-2}-r+c_{1} \cdot \beta \geqslant 0$, then necessarily $\gamma>0$. In this case we obtain an exponentially growing upper bound for $\operatorname{Ens}(t)$ with growth rate slightly larger than $-\nu \cdot c_{1}^{-2}-r+c_{1}$ $\beta$.

If, on the other hand, $-\nu \cdot c_{1}^{-2}-r+c_{1} \cdot \beta<0$, then we can choose $\gamma<0$. This furnishes $a$ polynomial upper bound which grows at least linearly in time. The precise growth exponent is determined by the regularity of the noise.

Remark 3: As we already stated in the beginning of this section, one can remove the condition of stochastic independence of $\omega_{0}$ and $W$ in the previous theorem, if one additionally assumes $\mathbb{E}\left\|\omega_{0}\right\|^{2+\delta}<\infty$ for some small $\delta$.

Our above results hold for a large class of noise processes, in particular also for more irregular Wiener processes $W$ whose covariance operator is not of trace class. If, however, one assumes that the Wiener process is of trace class, i.e., if $\operatorname{Tr}(Q)=\Sigma_{k=1}^{\infty} \mu_{k}^{2}<\infty$, then the results can be improved significantly by employing Ito's formula. One advantage of this approach is that it avoids the conditions on the eigenfunction in (7). Therefore, we will briefly outline the main ideas.

By applying Ito's formula (Ref. 16, Sec. 4.5) to the squared $L^{2}$-norm of the vorticity $\omega(t)$, it can easily be verified that

$$
\mathbb{E}\|\omega(t)\|^{2}=2 \mathbb{E} \int_{0}^{t}\langle A \omega(\tau)+F(\omega(\tau)), \omega(\tau)\rangle d \tau+\operatorname{Tr}(Q) \cdot t,
$$

where $\operatorname{Tr}(Q)=\sum_{k=1}^{\infty} \mu_{k}^{2}$ denotes the trace of the covariance operator $Q$ of $W$. Using calculations analogous to the ones leading to the a priori estimate in Lemma 1, we formally obtain

$$
\begin{aligned}
\partial_{t} \mathbb{E}\|\omega(t)\|^{2} & =2 \mathbb{E}\langle A \omega(\tau)+F(\omega(\tau)), \omega(\tau)\rangle+\operatorname{Tr}(Q) \\
& \leqslant 2 \gamma \cdot \mathbb{E}\|\omega(t)\|^{2}+\operatorname{Tr}(Q),
\end{aligned}
$$

where $\gamma>-\nu \cdot c_{1}^{-2}-r+c_{1} \cdot \beta$ as in Lemma 1 . Hence, for any $t \geqslant 0$ we have

$$
\operatorname{Ens}(t) \leqslant \operatorname{Ens}(0) \cdot e^{2 \gamma \cdot t}+\operatorname{Tr}(Q) \cdot \frac{e^{2 \gamma \cdot t}-1}{4 \gamma} .
$$

Especially if one can choose a growth exponent $\gamma<0$, this significantly improves the estimates of Theorem 2, since in this case the right-hand side of (18) approaches $-\operatorname{Tr}(Q) /(4 \gamma)$ for $t \rightarrow \infty$.

\section{ENSTROPHY ESTIMATE: HÖLDER CONTINUITY}

In this section we establish regularity properties of the enstrophy as a function of time. More precisely, we will prove that $\operatorname{Ens}(t)=\mathbb{E}\|\omega(t)\|^{2} / 2$ is Hölder continuous. To this end, we need the following lemma from Ref. 5 
Lemma 3: Define a nonlinear mapping $\mathcal{F}: C\left([0, T] ; H_{0}^{1}\right) \rightarrow C\left([0, T] ; L^{2}\right)$ by

$$
(\mathcal{F}(\omega))(t):=\int_{0}^{t} S(t-s) F(\omega(s)) d s, \quad \text { for } \quad t \in[0, T],
$$

where $\omega \in C\left([0, T] ; H_{0}^{1}\right)$, and $A$ and $F$ are as in $(9)$. Then $\mathcal{F}$ is continuous, and it can be extended to a continuous mapping from the space $C\left([0, T] ; L^{2}\right)$ to $C\left([0, T] ; L^{2}\right)$. Furthermore, the image of the extended mapping $\mathcal{F}$ is contained in $C\left([0, T], H^{a}(D)\right)$ for $0 \leqslant a<\frac{1}{2}$.

In fact, it is shown in Ref. 5 that for arbitrary positive constants $a \in\left[0, \frac{1}{4}\right)$ and $\rho \in\left(0, \frac{1}{4}\right)$ satisfying $0<\rho+a<\frac{1}{4}$ the estimate

$$
\begin{aligned}
&\left\|(-A)^{a} \int_{0}^{t} S(t-s) F(\omega(s)) d s\right\| \\
& \leqslant \frac{r c+\beta c}{1-a} \cdot t^{1-a} \cdot \sup _{0 \leqslant s \leqslant t}\|\omega(s)\| \\
&+\left(\frac{8 c}{1-4 \rho-4 a} \cdot t^{1 / 4-\rho-a}+\frac{4 c}{1-2 \rho-2 a} \cdot t^{1 / 2-\rho-a}\right) \cdot \sup _{0 \leqslant s \leqslant t}\|\omega(s)\|^{2}
\end{aligned}
$$

holds. Especially for $a=0$ we obtain

$$
\begin{aligned}
\|\mathcal{F}(\omega)(t)\|=\left\|\int_{0}^{t} S(t-s) F(\omega(s)) d s\right\| \leqslant & (r c+\beta c) \cdot t \cdot \sup _{0 \leqslant s \leqslant t}\|\omega(s)\| \\
& +\left(\frac{8 c}{1-4 \rho} \cdot t^{1 / 4-\rho}+\frac{4 c}{1-2 \rho} \cdot t^{1 / 2-\rho}\right) \cdot \sup _{0 \leqslant s \leqslant t}\|\omega(s)\|^{2},
\end{aligned}
$$

for every $0<\rho<\frac{1}{4}$. Together with Theorem 1 and Remark 1 these bounds immediately furnish the following result.

Lemma 4: Suppose that $\omega_{0}$ and $W$ are stochastically independent and that for some $p \geqslant 1$ we have $\mathbb{E}\left\|\omega_{0}\right\|^{2 p}<\infty$. Moreover, let $T>0$ be arbitrary, and let $a \in\left[0, \frac{1}{4}\right)$ and $\rho \in\left(0, \frac{1}{4}\right)$ be such that $0<\rho+a<\frac{1}{4}$. Then there exists a constant $C$ such that

$$
\mathbb{E}\left\|(-A)^{a} \mathcal{F}(\omega)(t)\right\|^{p} \leqslant C \cdot t^{p \cdot(1 / 4-\rho-a)} \quad \text { for all } t \in[0, T] .
$$

The following theorem states our main result on the regularity of the enstrophy. It will be proved in the remainder of this section.

Theorem 3 (Hölder continuity): Suppose that $\omega_{0}$ and $W$ are stochastically independent and that $\mathbb{E}\left\|\omega_{0}\right\|^{4}<\infty$. Then the enstrophy $\operatorname{Ens}(\cdot)$ defined by $\operatorname{Ens}(t)=\mathbb{E}\|\omega(t)\|^{2} / 2$ is Hölder continuous with arbitrary exponent less than $\frac{1}{4}$ on every compact interval in $(0, \infty)$.

Remark 4: Note that in general we cannot expect the solution $\omega$ to be Hölder continuous with arbitrary exponent less than $\frac{1}{4}$, since $W_{A}$ is in general less regular. As one can see from (10) with $\alpha=0$, one cannot expect $\omega$ to be more regular than $W_{A}$.

To prove the above theorem establishing the Hölder continuity of the enstrophy, we first define

$$
\mathcal{G}(\omega)(t):=S(t) \omega_{0}+\mathcal{F}(\omega)(t)
$$

According to (10) for $\alpha=0$, we therefore have $\omega(t)=\mathcal{G}(\omega)(t)+W_{A}(t)$. Consider a fixed interval $J=[\varepsilon, T] \subset(0, \infty)$. For $t \in J$ and $h$ with $t+h \in J$ the identity (10) then implies 


$$
\begin{aligned}
\mathbb{E}\|\omega(t+h)\|^{2}-\mathbb{E}\|\omega(t)\|^{2}= & \mathbb{E}\left\|\mathcal{G}(\omega)(t+h)+W_{A}(t+h)\right\|^{2}-\mathbb{E}\left\|\mathcal{G}(\omega)(t)+W_{A}(t)\right\|^{2} \\
= & \mathbb{E}\|\mathcal{G}(\omega)(t+h)\|^{2}-\mathbb{E}\|\mathcal{G}(\omega)(t)\|^{2}+2 \mathbb{E}\left\langle\mathcal{G}(\omega)(t+h), W_{A}(t+h)\right\rangle \\
& -2 \mathbb{E}\left\langle\mathcal{G}(\omega)(t), W_{A}(t)\right\rangle+\mathbb{E}\left\|W_{A}(t+h)\right\|^{2}-\mathbb{E}\left\|W_{A}(t)\right\|^{2} \\
= & : D_{1}+2 D_{2}+D_{3} .
\end{aligned}
$$

For simplicity we assume $h>0$ in the following. The case $h<0$ can be treated analogously.

We begin by estimating $D_{3}$. Due to Ref. 25 one has $\mathbb{E}\left\|W_{A}(\cdot)\right\|^{2} \in C^{\infty}(0, \infty)$. Hence, $\left|D_{3}\right|$ $\leqslant C \cdot h$ for some constant $C>0$.

In order to estimate $D_{2}$, we define the shift-operator $\tau_{t}$ by $\tau_{t} \omega=\omega(t+\cdot)$ for $t>0$. Then the definitions of $\mathcal{G}$ and $\mathcal{F}$ in (19) and Lemma 3, respectively, furnish

$$
\mathcal{G}(\omega)(t+h)=S(h) \mathcal{G}(\omega)(t)+\mathcal{F}\left(\tau_{t} \omega\right)(h) .
$$

Since $\{\omega(s)\}_{s \in[0, t]}$ is stochastically independent of $\{W(s)\}_{s \in[t, t+h]}$ we get

$$
\begin{aligned}
\mathbb{E}\left\langle\mathcal{G}(\omega)(t+h), W_{A}(t+h)\right\rangle & =\mathbb{E}\left\langle S(h) \mathcal{G}(\omega)(t), \int_{0}^{t} S(t+h-s) d W(s)\right\rangle+\mathbb{E}\left\langle\mathcal{F}\left(\tau_{t} \omega\right)(h), W_{A}(t+h)\right\rangle \\
& =\mathbb{E}\left\langle S(h) \mathcal{G}(\omega)(t), S(h) W_{A}(t)\right\rangle+\mathbb{E}\left\langle\mathcal{F}\left(\tau_{t} \omega\right)(h), W_{A}(t+h)\right\rangle
\end{aligned}
$$

and together with the self-adjointness of $S(h)$ we finally arrive at

$$
D_{2}=\mathbb{E}\left\langle(S(2 h)-I) \mathcal{G}(\omega)(t), W_{A}(t)\right\rangle+\mathbb{E}\left\langle\mathcal{F}\left(\tau_{t} \omega\right)(h), W_{A}(t+h)\right\rangle .
$$

The boundedness of $\mathbb{E}\left\|W_{A}(t)\right\|^{2}$ on $J$ and Lemma 4 now yield

$$
\left|\mathbb{E}\left\langle\mathcal{F}\left(\tau_{t} \omega\right)(h), W_{A}(t+h)\right\rangle\right| \leqslant\left(\mathbb{E}\left\|\mathcal{F}\left(\tau_{t} \omega\right)(h)\right\|^{2}\right)^{1 / 2} \cdot\left(\mathbb{E}\left\|W_{A}(t+h)\right\|^{2}\right)^{1 / 2} \leqslant C \cdot h^{1 / 4-\rho} .
$$

As for the first term in (21), notice that

$$
\|(S(h)-I) v\| \leqslant \int_{0}^{h}\|(-A) S(s) v\| d s \leqslant C \cdot h^{a} \cdot\left\|(-A)^{a} v\right\|
$$

for any $v \in D\left((-A)^{a}\right)$, with a constant $C$ which depends on $a \in[0,1)$. Thus,

$$
\begin{aligned}
& \left|\mathbb{E}\left\langle(S(2 h)-I) \mathcal{G}(\omega)(t), W_{A}(t)\right\rangle\right| \\
& \quad \leqslant C \cdot h^{1 / 4-\rho} \cdot\left(\mathbb{E}\left\|(-A)^{1 / 4-\rho} \mathcal{F}(\omega)(t)\right\|^{2}+\mathbb{E}\left\|(-A)^{1 / 4-\rho} S(t) \omega_{0}\right\|^{2}\right)^{1 / 2}
\end{aligned}
$$

for any $\rho \in\left(0, \frac{1}{4}\right)$. Together with $\left\|(-A)^{1 / 4-\rho} S(t) \omega_{0}\right\| \leqslant C \cdot \varepsilon^{\rho-1 / 4} \cdot\left\|\omega_{0}\right\|$ and Lemma 4 we eventually obtain

$$
\left|D_{2}\right| \leqslant C \cdot h^{1 / 4-\rho} .
$$

Finally we turn our attention to $D_{1}$. Its definition and (20) imply

$$
\begin{aligned}
D_{1} & =\mathbb{E}\|\mathcal{G}(\omega)(t+h)\|^{2}-\mathbb{E}\|\mathcal{G}(\omega)(t)\|^{2} \\
& =\mathbb{E}\langle\mathcal{G}(\omega)(t+h)-\mathcal{G}(\omega)(t), \underbrace{\mathcal{G}(\omega)(t+h)+\mathcal{G}(\omega)(t)}_{=: D_{\mathcal{G}}}\rangle \\
& =\mathbb{E}\left\langle(S(h)-I) \mathcal{G}(\omega)(t), D_{\mathcal{G}}\right\rangle+\mathbb{E}\left\langle\mathcal{F}\left(\tau_{t} \omega\right)(h), D_{\mathcal{G}}\right\rangle .
\end{aligned}
$$

As in the discussion leading to (23), we obtain for any $\widetilde{a} \in\left[0, \frac{1}{4}\right)$ 


$$
\left|\mathbb{E}\left\langle(S(h)-I) \mathcal{G}(\omega)(t), D_{\mathcal{G}}\right\rangle\right| \leqslant C \cdot h^{2 \tilde{a}} \cdot \mathbb{E}\left(\left\|(-A)^{\tilde{a}} \mathcal{G}(\omega)(t)\right\| \cdot\left\|(-A)^{\tilde{a}} D_{\mathcal{G}}\right\|\right) \leqslant C \cdot h^{2 \tilde{a}} .
$$

Using again Lemma 4 we further derive

$$
\left|\mathbb{E}\left\langle\mathcal{F}\left(\tau_{t} \omega\right)(h), D_{\mathcal{G}}\right\rangle\right| \leqslant C \cdot\left(\mathbb{E}\left\|\mathcal{F}\left(\tau_{t} \omega\right)(h)\right\|^{2}\right)^{1 / 2} \cdot\left(\mathbb{E}\|\| D_{\mathcal{G}} \|^{2}\right)^{1 / 2} \leqslant C \cdot h^{1 / 4-\rho} .
$$

Combining (24) for fixed $\widetilde{a}$ near $\frac{1}{4}$ with (25) furnishes $\left|D_{1}\right| \leqslant C \cdot h^{1 / 4-\rho}$, and we finally obtain

$$
|\operatorname{Ens}(t+h)-\operatorname{Ens}(t)| \leqslant C \cdot h^{1 / 4-\rho}
$$

for arbitrary $\rho \in\left(0, \frac{1}{4}\right)$. This completes the proof of Theorem 3 .

\section{ENSTROPHY ESTIMATE: ASYMPTOTICS}

In Sec. III we established upper bounds on the growth of the enstrophy $\operatorname{Ens}(t)$ $=\mathbb{E}\|\omega(t)\|^{2} / 2$. Unfortunately, these bounds fail to accurately describe the dynamics of $\operatorname{Ens}(t)$ as $t \rightarrow 0$. For example, the bound derived in Theorem 1 will generally not even converge to $\operatorname{Ens}(0)$ as $t \rightarrow 0$. Therefore, this section is devoted to investigating the small-time asymptotics of the enstrophy. Similar to Ref. 25 and 26 this will be accomplished by relating Ens $(t)$ to the stochastic convolution.

In order to bound the growth of $\mathbb{E}\left\|W_{A}(t)\right\|^{2}$, we assume that the coefficients $\mu_{k}$ in (6) are bounded by $\mu_{k}^{2} \leqslant c_{\mu} \cdot k^{-\delta}$ for some $\delta \in(0,1)$ and some positive constant $c_{\mu}>0$. In this situation we obtain similar to Ref. 25, Theorem 5.4, the estimate

$$
\mathbb{E}\left\|W_{A}(t)\right\|^{2} \leqslant C_{0} \cdot t^{\delta}
$$

for arbitrary $t \in[0, T]$, where $C_{0}$ denotes a positive constant which depends on $T$. Using the mild integral form (10) we further get

$$
\left\|\omega(t)-\omega_{0}-W_{A}(t)\right\| \leqslant\left\|(S(t)-I) \omega_{0}\right\|+\|\mathcal{F}(\omega)(t)\| .
$$

If we now assume that $\mathbb{E}\left\|\omega_{0}\right\|^{4}<\infty$, then an application of Lemma 4 furnishes

$$
\begin{aligned}
\mathbb{E}\left\|\omega(t)-\omega_{0}-W_{A}(t)\right\|^{2} & \leqslant 2 \mathbb{E}\left\|(S(t)-I) \omega_{0}\right\|^{2}+2 \mathbb{E}\|\mathcal{F}(\omega)(t)\|^{2} \\
& \leqslant 2 \mathbb{E}\left\|(S(t)-I) \omega_{0}\right\|^{2}+C \cdot t^{1 / 2-2 \rho} \\
& \leqslant C \cdot t^{2 \gamma} \cdot \mathbb{E}\left\|(-A)^{\gamma} \omega_{0}\right\|^{2}+C \cdot t^{1 / 2-2 \rho}
\end{aligned}
$$

for fixed $\rho \in\left(0, \frac{1}{4}\right)$ and $\gamma \in[0,1)$. Thus, the additional assumption $\mathbb{E}\left\|(-A)^{\gamma} \omega_{0}\right\|^{2}<\infty$ for some small $\gamma \in[0,1)$ implies

$$
\mathbb{E}\left\|\omega(t)-\omega_{0}-W_{A}(t)\right\|^{2} \leqslant C \cdot t^{2 \gamma}
$$

Hence,

$$
\begin{aligned}
\left(\mathbb{E}\|\omega(t)\|^{2}\right)^{1 / 2} & \leqslant\left(\mathbb{E}\left\|\omega_{0}\right\|^{2}\right)^{1 / 2}+\left(\mathbb{E}\left\|W_{A}(t)\right\|^{2}\right)^{1 / 2}+\left(\mathbb{E}\left\|\omega(t)-\omega_{0}-W_{A}(t)\right\|^{2}\right)^{1 / 2} \\
& =\left(\mathbb{E}\left\|\omega_{0}\right\|^{2}\right)^{1 / 2}+\mathcal{O}\left(t^{\gamma}+t^{\delta / 2}\right) .
\end{aligned}
$$

Similarly one obtains

$$
\begin{aligned}
\left(\mathbb{E}\|\omega(t)\|^{2}\right)^{1 / 2} & \geqslant\left(\mathbb{E}\left\|\omega_{0}\right\|^{2}\right)^{1 / 2}-\left(\mathbb{E}\left\|W_{A}(t)\right\|^{2}\right)^{1 / 2}-\left(\mathbb{E}\left\|\omega(t)-\omega_{0}-W_{A}(t)\right\|^{2}\right)^{1 / 2} \\
& =\left(\mathbb{E}\left\|\omega_{0}\right\|^{2}\right)^{1 / 2}+\mathcal{O}\left(t^{\gamma}+t^{\delta / 2}\right),
\end{aligned}
$$

and together these estimates show that $\mathbb{E}\|\omega(t)\|^{2}=\mathbb{E}\left\|\omega_{0}\right\|^{2}+\mathcal{O}\left(t^{\gamma}+t^{\delta / 2}\right)$. If, on the other hand, we have $\omega_{0}=0$, then (27) implies 


$$
\mathbb{E}\left\|\omega(t)-W_{A}(t)\right\|^{2} \leqslant t^{1 / 2-2 \rho}
$$

which analogously results in $\mathbb{E}\|\omega(t)\|^{2}=\mathbb{E}\left\|W_{A}(t)\right\|^{2}+\mathcal{O}\left(t^{\frac{1}{4}}-\rho+\delta / 2\right)$. Using the definition of Ens, this furnishes the following result on the small-time asymptotics of the enstrophy.

Theorem 4(asymptotics): Assume that $\mathbb{E}\left\|(-A)^{\gamma} \omega_{0}\right\|^{2}<\infty$ for some small constant $\gamma>0$ and that $\mathbb{E}\left\|\omega_{0}\right\|^{4}<\infty$. Furthermore, suppose that (26) holds for some small $\delta>0$. Then

$$
\operatorname{Ens}(t)=\frac{1}{2} \cdot \mathbb{E}\left\|\omega_{0}\right\|^{2}+\mathcal{O}\left(t^{\gamma}+t^{\delta / 2}\right)
$$

If in addition we have $\omega_{0}=0$ and let $\rho \in\left(0, \frac{1}{4}\right)$ be arbitrary, then

$$
\operatorname{Ens}(t)=\frac{1}{2} \cdot \mathbb{E}\left\|W_{A}(t)\right\|^{2}+\mathcal{O}\left(t^{(1+2 \delta) / 4-\rho}\right) .
$$

Notice that in the case $\omega_{0}=0$ the second term on the right-hand side is of higher order than $\mathbb{E}\left\|W_{A}(t)\right\|^{2} / 2$ only under additional assumptions. For this we need $\delta<\frac{1}{2}-2 \rho$, as well as a suitable lower bound on the growth of the first term $\mathbb{E}\left\|W_{A}(t)\right\|^{2} / 2$ for small values of $t$. The latter can be achieved by imposing a lower bound on the growth of the coefficients $\mu_{k}$. For details we refer the reader to Refs. 25 and 27.

\section{SUMMARY}

The enstrophy $\operatorname{Ens}(t)=\mathbb{E}\|\omega(t)\|^{2} / 2$ is an averaged measure of fluid vorticity $\omega(t)$. We have investigated the enstrophy evolution of large-scale quasi-geostrophic flows under random wind forcing. Thereby we have obtained results on upper bounds (Theorems 1 and 2), Hölder continuity (Theorem 3), as well as small-time asymptotics (Theorem 4) for the enstrophy.

\section{ACKNOWLEDGMENTS}

A part of this work was done at the Institute of Mathematics and Its Applications, Minnesota, and the Oberwolfach Mathematical Research Institute, Germany, while J.D. was a Research in Pairs Fellow, supported by Volkswagen Stiftung. This work was partly supported by the NSF Grant No. DMS-9973204.

\footnotetext{
${ }^{1}$ P. Müller, "Stochastic forcing of quasi-geostrophic eddies," in Stochastic Modelling in Physical Oceanography, edited by R. J. Adler, P. Müller, and B. Rozovskii (Birkhäuser, Boston, 1996).

${ }^{2}$ G. Holloway, "Ocean circulation: Flow in probability under statistical dynamical forcing," in Stochastic Models in Geosystems, edited by S. Molchanov and W. Woyczynski (Springer, New York, 1996).

${ }^{3}$ A. Griffa and S. Castellari, J. Mar. Res. 49, 53 (1991).

${ }^{4}$ R. M. Samelson, J. Geophys. Res. 94, 8207 (1989).

${ }^{5}$ J. Brannan, J. Duan, and T. Wanner, J. Math. Anal. Appl. 228, 221 (1998).

${ }^{6}$ P. Sura, K. Fraedrich, and F. Lunkeit, J. Phys. Oceanogr. 30, 1917 (2000).

${ }^{7}$ P. Sura, K. Fraedrich, and F. Lunkeit, J. Phys. Oceanogr. 31, 411 (2001).

${ }^{8}$ J. Brannan, J. Duan, and V. Ervin, Physica D 133, 23 (1999).

${ }^{9}$ J. Brannan, J. Duan, and V. Ervin, Math. Prob. Eng. 7, 55 (2001).

${ }^{10}$ D. Szurley and J. Duan, Appl. Math. Comput. 118, 261 (2001).

${ }^{11}$ J. Duan and B. Schmalfuss, Physica D (submitted).

${ }^{12}$ J. Pedlosky, Ocean Circulation Theory (Springer, New York, 1996).

${ }^{13}$ V. Dymnikov and E. Kazantsev, "On the genetic "memory' of chaotic attractor of the barotropic ocean model," in Proceedings of the Third Bilateral Conference "Predictability of Atmospheric and Oceanic Circulations" of the FrenchRussian A.M. Liapunov Institute in Computer Science and Applied Mathematics (INRIA-Moscow State University). Nancy, April 1997, MSU edition, pp. 25-36.

${ }^{14}$ J. Pedlosky, Geophysical Fluid Dynamics, 2nd ed. (Springer, New York, 1987).

${ }^{15}$ R. Salmon, Lectures on Geophysical Fluid Dynamics (Oxford University Press, New York, 1998).

${ }^{16}$ G. Da Prato and J. Zabczyk, Stochastic Equations in Infinite Dimensions (Cambridge University Press, Cambridge, 1992).

${ }^{17}$ D. Gilbarg and N. S. Trudinger, Elliptic Partial Differential Equations of Second Order, 2nd ed. (Springer, New York, 1983).

${ }^{18}$ A. Pazy, Semigroups of Linear Operators and Applications to Partial Differential Equations (Springer, New York, 1983). ${ }^{19}$ R. Aurich, A. Bäcker, R. Schubert, and M. Taglieber, Physica D 129, 1 (1999).

${ }^{20}$ G. Da Prato and J. Zabczyk, Ergodicity for Infinite Dimensional Systems (Cambridge University Press, Cambridge, 1996).
} 
${ }^{21}$ J. K. Hale, Ordinary Differential Equations, 2nd ed. (Krieger, Malabar, 1980).

${ }^{22}$ C. Gugg, "Approximation of Stochastic Partial Differential Equations and Turbulence in Fluids," Ph.D. thesis, Universität Augsburg, 2000.

${ }^{23}$ C. Gugg, H. Kielhöfer, and M. Niggemann, "On the approximation of the stochastic Burgers equation," preprint (2001).

${ }^{24}$ D. E. Edmunds and W. D. Evans, Spectral Theory and Differential Operators (Oxford Science, New York, 1990).

${ }^{25}$ D. Blömker, S. Maier-Paape, and T. Wanner, Interfaces Free Boundaries 3, 465 (2001).

${ }^{26}$ D. Blömker, S. Maier-Paape, and T. Wanner, Stochastics Dynam. 1, 239 (2001).

${ }^{27}$ J. Duan and B. Goldys, Int. J. Math. Math. Sci. (in press). 